Tropical Journal of Pharmaceutical Research April 2021; 20 (4): 815-823

ISSN: $1596-5996$ (print); 1596-9827 (electronic)

(C) Pharmacotherapy Group, Faculty of Pharmacy, University of Benin, Benin City, 300001 Nigeria.

Available online at http://www.tjpr.org

Original Research Article

http://dx.doi.org/10.4314/tjpr.v20i4.23

\title{
Molecular docking and dynamic simulation study to explore quercetin as a multi-potent candidate against gliomas
}

\author{
Syed Mohd Danish Rizvi ${ }^{1}$, Talib Hussain², Khalid Mehmood ${ }^{1,3}$, Afrasim Moin ${ }^{1 *}$, \\ Abulrahman Sattam Alanazi ${ }^{4}$, Gehad M Subaiea ${ }^{2}$ \\ ${ }^{1}$ Department of Pharmaceutics, ${ }^{2}$ Department of Pharmacology and Toxicology, College of Pharmacy, University of Hail, Hail, \\ Saudi Arabia, ${ }^{3}$ Department of Pharmacy, Abbottabad University of Science and Technology, Havelian, Pakistan, ${ }^{4}$ Department \\ of Clinical Pharmacy, College of Pharmacy, University of Hail, Hail, Saudi Arabia
}

*For correspondence: Email: afrasimmoin@yahoo.co.in

Sent for review: 14 December 2020

Revised accepted: 21 March 2021

\begin{abstract}
Purpose: To search for novel gliomas targets and their inhibitors using a molecular docking approach. Methods: Quercetin multi-targeting potential was investigated against some of the emerging gliomas targets such as epidermal growth factor receptor (EGFR), ephrin type-A receptor 2 (EphA2), nicotinamide phosphoribosyltransferase (NMRPTase) and plasminogen activator inhibitor-1 (PAl-1). Crucial biochemical interaction of quercetin with these targets were analyzed using molecular docking study.

Results: Quercetin interacted strongly via hydrogen bonding with important active sites consisting of amino acid residues of EphA2 and PAl-1, and showed binding energy of -7.44 and $-7.38 \mathrm{kcal} / \mathrm{mol}$, respectively. Some crucial active site amino acids involved in the interaction of known EphA2 and PAl-1 inhibitors (Alw-Il-41-27 and ACT001) were common in quercetin interactions as well, and both inhibitors as well as quercetin did not violate Lipinski rules. Importantly, the quercetin-EphA2 and quercetin-PAI-1 complexes were stable as minimal fluctuations within the permissible limit were observed during a $20 \mathrm{~ns}$ trajectory performed on desmond simulation platform.

Conclusion: Despite the fact that quercetin has been studied extensively against various cancer pathways, its transformation from a long-time bench candidate into bedside medications still needs further exploration. Nevertheless, the present predictive biochemical interaction analysis against emerging glioma targets might pave way for the design of novel therapeutic agents based on quercetin scaffolds.
\end{abstract}

Keywords: Ephrin type-A receptor 2, Gliomas, Molecular docking, Plasminogen activator inhibitor-1, Quercetin, Simulation analysis

This is an Open Access article that uses a fund-ing model which does not charge readers or their institutions for access and distributed under the terms of the Creative Commons Attribution License (http://creativecommons.org/licenses/by/4.0) and the Budapest Open Access Initiative (http://www.budapestopenaccessinitiative.org/read), which permit unrestricted use, distribution, and reproduction in any medium, provided the original work is properly credited.

Tropical Journal of Pharmaceutical Research is indexed by Science Citation Index (SciSearch), Scopus, International Pharmaceutical Abstract, Chemical Abstracts, Embase, Index Copernicus, EBSCO, African Index Medicus, JournalSeek, Journal Citation Reports/Science Edition, Directory of Open Access Journals (DOAJ), African Journal Online, Bioline International, Open-J-Gate and Pharmacy Abstracts

\section{INTRODUCTION}

Glioblastoma (GBM) is considered a very common and highly aggressive primary malignant tumor of the brain in adult populations and it is often associated with poor patient prognosis and high morbidity/mortality [1]. Like most of the tumors, the standard therapeutic intervention for GBM involves several sessions of radio- 
chemotherapy, followed by surgical removal of the tumor [2]. However, potential resistance against postoperative radiochemotherapy in GBM cases generally reduces the patient survival. To overcome the problems of severe side effects and resistance associated with conventional chemotherapy and consequently improving patient survival rate, glioma treatment warrants new therapeutic interventions including but not limited to development of novel chemotherapeutic agents and/or new combinations of the existing drugs.

Natural compounds constitute major sources for the development of novel drugs, and their ability to cross-blood-brain barrier (BBB) makes them as more interesting candidates. Quercetin (3, 30, 40, 5, 7-pentahydroxy flavone) is a natural polyphenolic flavonoid, which is present in several fruits and vegetables. The chemopreventive effects of quercetin have been investigated in various in vitro and in vivo cancer models. Moreover, quercetin has been associated with tumor growth reduction in breast, prostate and colon [3-5].

Quercetin can induce apoptosis in tumour cells, and shows interaction with cyclindependent kinase (CDK)-4 and cyclin D1, the regulatory proteins of cell cycle that leads to activation of $p 53$, induction of caspase-3 and caspase-9, and release of cytochrome c [6]. Furthermore, it has been suggested as a multi-target agent due to its $\mathrm{PI} K$ inhibition potential that results in suppression of tumor growth [7]. It has also been established that aglycone quercetin is associated with production of several pro-inflammatory mediators and microglia proliferation. In addition, aglycone quercetin can be used as a possible adjuvant in treatment regimens owing to its modulatory effects on microglia profile [8].

Studies involving animal (including human) and cellular models have suggested that quercetin exerts its antioxidant action through scavenging the reactive oxygen and nitrogen species whereas antitumor and antiinflammatory effects employ modulation of gene expression and signaling pathways [9].

In the present study, quercetin potential was explored against some emerging therapeutic targets of gliomas, i.e., epidermal growth factor receptor, ephrin type-a receptor 2 , nicotinamide phosphoribosyltransferase and plasminogen activator inhibitor-1. The computational biology approach including molecular dynamics simulation analysis was used in the present study to predict the binding interaction of quercetin with gliomas targets and their stability analysis.

\section{METHODS}

\section{Quercetin and target proteins 3D structures retrieval from databases}

Three dimensional structures of gliomas target proteins in pdb format were retrieved from Protein Data Bank. The PDB ID for target proteins epidermal growth factor receptor (EGFR), ephrin type-A receptor 2 (EphA2), nicotinamide phosphoribosyltransferase (NMRPTase) and plasminogen activator inhibitor-1 (PAl-1) were 5FED, 1MQB, 2GVG and 4G8R, respectively. However, 3-D structure of ligand Quercetin (CID: 5280343) was fetched from PubChem database.

\section{Molecular docking interaction study}

Rizvi et al protocol was applied for molecular docking interaction study using 'Autodock4.2' [10]. The MMFF94 force field was applied for energy minimization of quercetin. Hydrogen atoms, solvation constrains and Kollman charges were added through AutoDock. Grid maps with specific co-ordinates were set using Autogrid tool for docking quercetin precisely into the active site of each glioma target protein. Grid points were kept as $80 \times$ $60 \times 50 \AA$ for NMRPTase and $60 \times 60 \times 60 \AA$ for EGFR, EphA2 and PAl-1 with $0.375 \AA$ spacing. However, the $x, y$ and $z$ co-ordinates for EGFR, EphA2, NMRPTase and PAl-1, were set as '0.232, 53.667 and -18.508', '44.712, -3.967 and 85.623', '30.311, 3.397 and 45.860' and '-35.219, -20.703 and 31.925 ', respectively. Solis \& Wets local search and Lamarckian genetic algorithm approach were used for docking each of these proteins with quercetin. Hundred different runs were used for each docking with an end limit of 2,500,000 energy assessments.

\section{LIGPLOT+ analysis for each docked structure}

LIGPLOT+ Version v.1.4.5 was used to analyze each docked complex [11]. LIGPLOT analysis helped in examining the hydrophobic interactions and hydrogen bonds among quercetin and different glioma target proteins. Moreover, LIGPLOT algorithm was used to 
transform 3-D structures for each interaction into final 2-D figures.

\section{Molecular dynamic (MD) simulation study}

Molecular dynamic (MD) simulation was executed to reveal the stability and effectiveness of best-docked complexes, i.e., 'EphA2-Quercetin' and 'PAl-1-Quercetin'. Desmond package (Schrödinger Release 2020) with OPLS3 force-field was used for the simulation. The solvation of molecular system was done with water molecules and the charge of the system was neutralized using $\mathrm{Na}+/ \mathrm{Cl}$ - ions. Prior to MD simulation, system energy was minimized by heating. However, minimization for each complex was also performed by minimization method via applying steepest descent steps-based protocol. Furthermore, the equilibrium condition at 1000 steepest descent steps for the system was maintained and updated. After system equilibration, simulation was run for 20ns (at 1atm pressure and 300k temperature) using the NPT-ensembles.

\section{RESULTS}

In the present study, quercetin was first docked with epidermal growth factor receptor (EGFR) to investigate its potential. The molecular docking interaction of 'Quercetin' with 'EGFR' revealed the involvement of twelve amino acid residues, i.e., Leu718, Val726, Ala743, Lys745, Thr790, Gln791, Leu792, Met793, Gly796, Cys797, Asp800 and Leu844 (Table 1). Moreover, ligplot analysis showed that Lys745, Thr790, GIn791, Met793 and Cys797 amino acid residues of 'EGFR active site' were involved in hydrogen bonding, whereas, Leu718, Val726, Ala743, Leu792, Gly796 and Leu844 amino acid residues exhibited hydrophobic interactions with quercetin (Figure 1).

Binding energy $(\Delta G)$ and inhibition constant (Ki) for 'EGFR-Quercetin' complex were found to be $-6.73 \mathrm{kcal} / \mathrm{mol}$ and $11.62 \mu \mathrm{M}$, respectively (Table 1). The 'electrostatic energy', 'total Van der Waals, Hydrogen bond and desolvation energy', and 'total intermolecular energy' of quercetin interaction with EGFR were estimated to be $-0.16,-8.36$ and $-8.52 \mathrm{kcal} / \mathrm{mol}$, respectively.

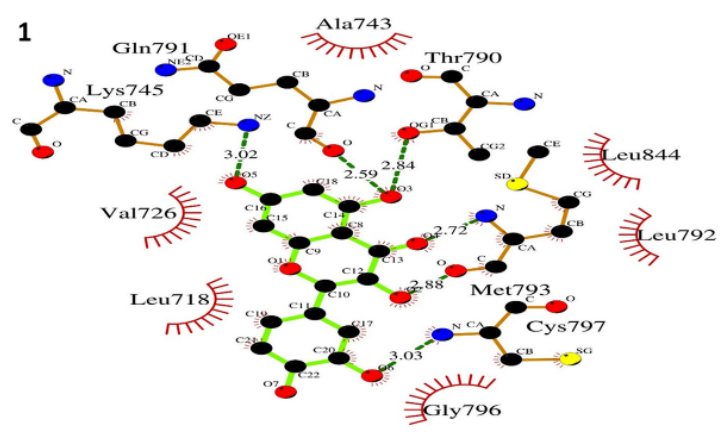

Figure 1: LigPlot of 'Quercetin-EGFR' interactions. Here, the hydrophobic interacting amino acid residues are shown as red arcs, and hydrogen bond as green dashed line

Second target of the present study was EphA2. The docking analysis showed that 16 amino acid residues of 'active site of EphA2' were involved in interactions with quercetin (Table 1). Out of 16 the amino acids, six amino acid residues, namely, Glu663, Thr692, Glu693, Met695, Ser756 and Asp757 showed hydrogen bonding with quercetin. However, seven amino acid residues, i.e., Ile619, Ala644, Lys646, Met667, Tyr694, Gly698 and Leu746 interacted with quercetin via hydrophobic interaction (Figure 2).

Table 1: Molecular interaction of quercetin with different gliomas targets

\begin{tabular}{|c|c|c|c|}
\hline Target & Binding energy & $\mathbf{K i}$ & Amino acids \\
\hline$E G F R$ & $-6.73 \mathrm{kcal} / \mathrm{mol}$ & $11.62 \mathrm{nM}$ & $\begin{array}{l}\text { Leu718, Val726, Ala743, Lys745, } \\
\text { Thr790, GIn791, Leu792, Met793, } \\
\text { Gly796, Cys797, Asp800, Leu844 }\end{array}$ \\
\hline EphA2 & $-7.44 \mathrm{kcal} / \mathrm{mol}$ & $3.52 \mathrm{nM}$ & $\begin{array}{l}\text { Ile619, Gly620, Ala644, Lys646, } \\
\text { Glu663, Met667, Ile676, Thr692, } \\
\text { Glu693, Tyr694, Met695, Glu696, } \\
\text { Gly698, Leu746, Ser756, Asp757 }\end{array}$ \\
\hline NMRPTase & $-6.39 \mathrm{kcal} / \mathrm{mol}$ & $20.82 n M$ & $\begin{array}{l}\text { Phe193, Arg196, Gly353, Asp354, } \\
\text { Gly355, Val356, Asp357, Gly381, } \\
\text { Ser382, Gly383, Gly384, Gly385, } \\
\text { Lys389 }\end{array}$ \\
\hline PAl-1 & $-7.38 \mathrm{kcal} / \mathrm{mol}$ & 3.89nM & $\begin{array}{l}\text { Ala40, Ser41, Ala44, Met45, Ala72, } \\
\text { Leu75, Arg76, Tyr79, Thr93, Thr94, } \\
\text { Asp95, Leu116, Phe117, Ser119 }\end{array}$ \\
\hline
\end{tabular}


The $\Delta \mathrm{G}$ and $\mathrm{Ki}$ of EphA2-quercetin interaction were $-7.44 \mathrm{kcal} / \mathrm{mol}$ and $3.52 \mu \mathrm{M}$, respectively. The 'Van der Waals + Hbond+ desolvation' energy and 'Electrostatic' energy for the EphA2-quercetion complex were found to be $-8.89 \mathrm{kcal} / \mathrm{mol}$ and $-0.34 \mathrm{kcal} / \mathrm{mol}$, respectively, and the total intermolecular energy was $-9.23 \mathrm{kcal} / \mathrm{mol}$.

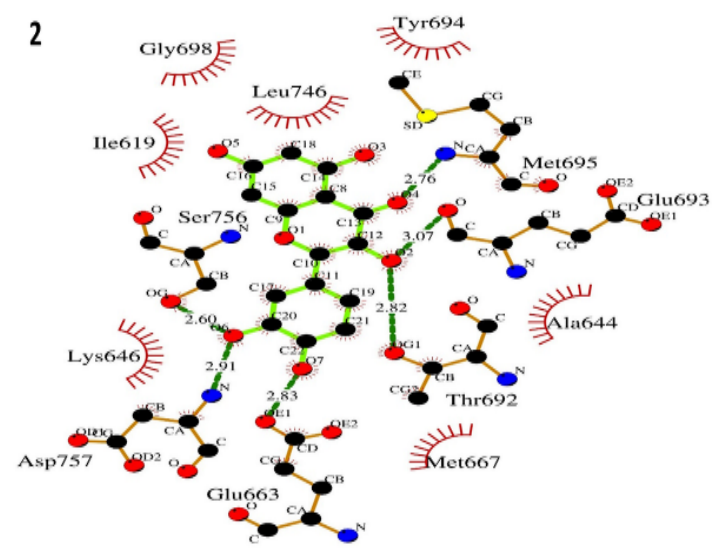

Figure 2: LigPlot of 'Quercetin-EphA2' interactions. Here, the hydrophobic interacting amino acid residues are shown as red arcs and hydrogen bond as green dashed line

Third target for the present study was NMPRTase. NMRPTase interaction with quercetin showed involvement of thirteen amino acid residues of active site of NMRPTase (Table 1). Three amino acid residues, namely, Val356, Gly381 and Lys389 showed strong hydrogen bonding, while, Phe193, Gly353, Gly355, Ser382, Gly383, Gly384 and Gly385 showed hydrophobic interaction with quercetin (Figure 3). The $\Delta \mathrm{G}$ and $\mathrm{Ki}$ of 'NMRPTase-Quercetin' interaction was found to be as $-6.39 \mathrm{kcal} / \mathrm{mol}$ and $20.82 \mu \mathrm{M}, \quad$ respectively. The Electrostatic, Van der Waals + Hbond+ desolvation energy and Total intermolecular energy for the 'NMRPTase-Quercetin' complex were found to be $-0.51 \mathrm{kcal} / \mathrm{mol}$, $7.67 \mathrm{kcal} / \mathrm{mol}$ and $-8.18 \mathrm{kcal} / \mathrm{mol}$, respectively.

The final and fourth target of the present study was PAl-1. The molecular interaction of 'PAI-1' with Quercetin showed involvement of 14 amino acid residues (Table 1). LigPlot analysis showed that two amino acid residues (Thr93 and Phe117) of PAl-1 interacted with hydrogen bonding, whereas, Ser41, Ala44, Met45, Leu75, Arg76, Tyr79, Thr94, Asp95 and Ser119 amino acids demonstrated hydrophobic interactions with quercetin (Figure 4). Moreover, the 'PAI-1' interaction with Quercetin showed $\Delta \mathrm{G}$ and $\mathrm{Ki}$ as $-7.38 \mathrm{kcal} / \mathrm{mol}$ and $3.89 \mu \mathrm{M}$, respectively. The total intermolecular, Electrostatic and Van der Waals + Hbond+ desolvation energy of quercetin binding with PAl-1 were -9.17 $\mathrm{kcal} / \mathrm{mol},-0.29 \mathrm{kcal} / \mathrm{mol}$ and $-8.88 \mathrm{kcal} / \mathrm{mol}$, respectively.

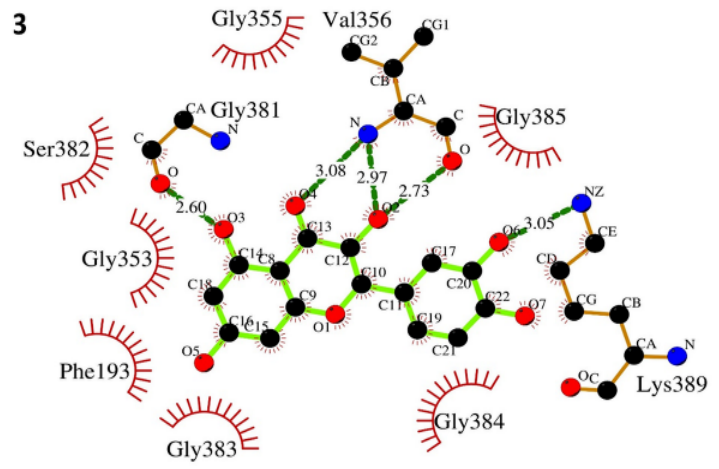

Figure 3: LigPlot of 'Quercetin-NMRPTase' interactions. Here, the hydrophobic interacting amino acid residues are shown as red arcs and hydrogen bond as green dashed line

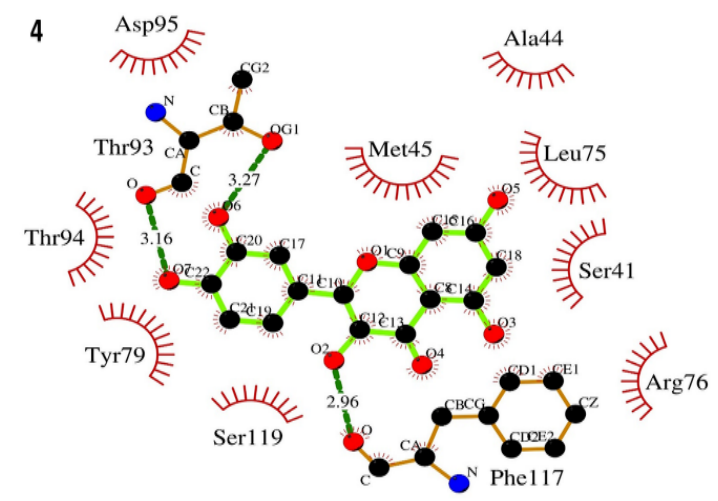

Figure 4: LigPlot of 'Quercetin-PAI-1' interactions. Here, the hydrophobic interacting amino acid residues are shown as red arcs and hydrogen bond as green dashed line

Based on the results, EphA2 and PAI-1 were selected as potential quercetin targets for further analysis. For comparative evaluation, EphA2 was further docked with its control 'Alw-II-41-27' and PAI-1 was docked with control 'ACT001' (Table 2). EphA2 interaction with Alw-II-41-27 showed $\Delta \mathrm{G}$ and $\mathrm{Ki}$ values $8.95 \mathrm{kcal} / \mathrm{mol}$ and $275.10 \mathrm{nM}$, respectively that is better than EphA2-Quercetin interaction. However, amino acid residues of EphA2 (Ile619, Gly620, Ala644, Tyr694, Met695, Glu696, Gly698, Leu746, Asp757) were common in interaction with control and quercetin. 
Table 2: Molecular interactions of control inhibitors with EphA2 and PAI-1

\begin{tabular}{|c|c|c|c|}
\hline Complex & Binding energy & $\mathbf{K i}$ & Amino acids \\
\hline $\begin{array}{l}\text { 'EphA2' and } \\
\text { 'Alw-II-41-27' }\end{array}$ & -8.95 & 275.10nM & $\begin{array}{l}\text { Ile619, Gly620, Ala621, Gly622, Glu623, Val627, Ala644, } \\
\text { Tyr694, Met695, Glu696, Asn697, Gly698, Asp739, } \\
\text { Leu746, Asp757, Phe758, Gly759, Leu760 }\end{array}$ \\
\hline $\begin{array}{l}\text { 'PAI-1' and } \\
\text { 'ACT001' }\end{array}$ & -6.82 & $10.04 \mathrm{nM}$ & $\begin{array}{l}\text { Tyr37, Ser41, Met45, Leu75, Arg76, Tyr79, Thr93, Thr94, } \\
\text { Asp95, Phe117, Ser119, Trp139 }\end{array}$ \\
\hline
\end{tabular}

Table 3: Physicochemical parameters of quercetin and control inhibitors

\begin{tabular}{lllllll}
$\begin{array}{l}\text { Compound } \\
\text { Name }\end{array}$ & $\begin{array}{l}\text { Molecular } \\
\text { Weight }\end{array}$ & cLogP & $\begin{array}{l}\text { Hydrogen } \\
\text { Bond Donors }\end{array}$ & $\begin{array}{l}\text { Hydrogen Bond } \\
\text { Acceptors }\end{array}$ & $\begin{array}{l}\text { Number of } \\
\text { Rotatable } \\
\text { Bonds }\end{array}$ & $\begin{array}{l}\text { Lipinski's } \\
\text { Violation }\end{array}$ \\
\hline Rule & $<500$ & $\leq 5$ & $<5$ & $<10$ & $\leq 10$ & $\leq 1$ \\
\hline Quercetin & 302.23 & 1.49 & 5 & 7 & 1 & 0 \\
\hline $\begin{array}{l}\text { Alw-II-41- } \\
27\end{array}$ & 609.71 & 4.74 & 2 & 7 & 9 & 1 \\
\hline ACT001 & 293.40 & 1.23 & 1 & 4 & 2 & 0 \\
\hline$* *$ Logarithm of compound partition coefficient between n-octanol and water
\end{tabular}

In contrast, PAl-1 interaction with ACT001 showed $\triangle G$ and $\mathrm{Ki}$ values $-6.82 \mathrm{kcal} / \mathrm{mol}$ and $10.04 \mu \mathrm{M}$, respectively that is lower than PAI1 and quercetin interaction. In addition, Ser41, Met45, Leu75, Arg76, Tyr79, Thr93, Thr94, Asp95, Phe117 and Ser119 were the common interacting amino acid residues of PAl-1 when interacted with control as well as quercetin. Moreover, none of the control compounds and quercetin violate the Lipinski's five rules (Table 3 ). These results prompted us to further analyse the EphA2Quercetin and PAl-1-Quercetin complexes via molecular dynamics (MD) simulation studies.

To estimate the stability of 'EphA2-Quercetin' and 'PAl-1-Quercetin' complexes, the dynamic pattern of the system was simulated using real time MD with OPLS3 force field via Desmond. EphA2 and PAl-1 protein frames were initially aligned on the reference backbone followed by root mean square deviation (RMSD) calculation on the basis of atomic selection. RMSD observation provided the detailed structural conformation during the simulation. RMSD plot with 20ns trajectory (Figure 5) indicated RMSD of target protein (EphA2) on left Y-axis, whereas, ligand (quercetin) RMSD profile aligned on protein backbone on the right $Y$-axis. Interestingly, during simulation the 'EphA2Quercetin' complex got stabilized with regard to reference frame at time Ons. Initially, from $0-4 \mathrm{~ns}$, the quercetin and protein backbone were almost laying over each other, and after some fluctuations quercetin and protein backbone again overlapped from 7.5 to $14 \mathrm{~ns}$. However, minimal fluctuations within the permissible limit of $1-3 \AA$ were observed during the entire 20ns simulation trajectory that could be ignored and considered nonsignificant. MD simulation results predicted that quercetin stably bound to EphA2 active sites without causing any large conformational change in the protein and without getting diffused away from the binding sites.

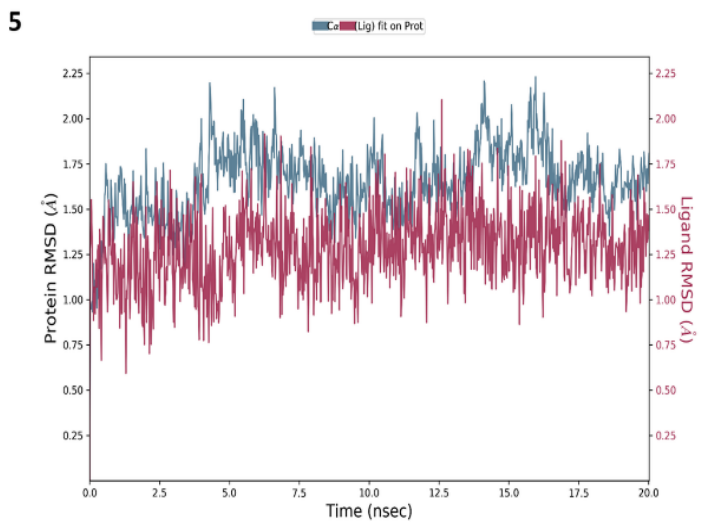

Figure 5: RMSD plot of MD simulation 20ns trajectory obtained for 'Quercetin-EphA2' complex

The similar pattern was observed in RMSD analysis of 'PAl-1-Quercetin' complex (Figure 6). Quercetin and PAI-1 protein showed initial fluctuations from 0 to $5 \mathrm{~ns}$ that were minimized later on from 5 to $10 \mathrm{~ns}$, and after 10ns RMSD was stable with overlapping of Quercetin and PAl-1 protein that implicated minimal conformational alterations in PAI-1 protein backbone. Nevertheless, fluctuation under the permissible limit was evident during the entire simulation process for 20ns trajectory inferring the stability of the 'PAI-1Quercetin' complex. 
6

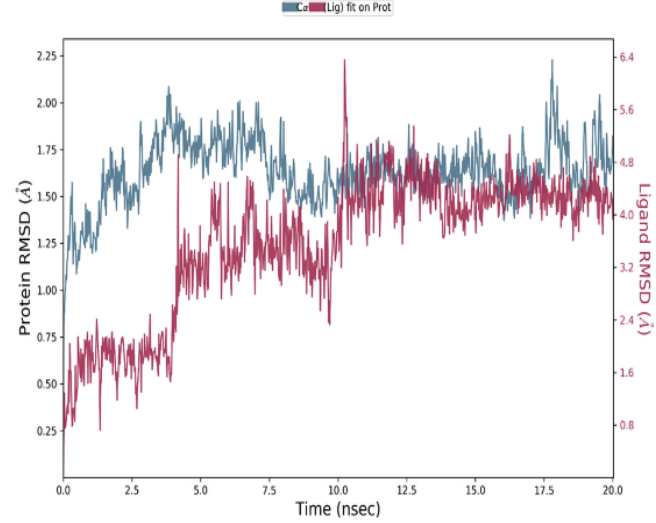

Figure 6: RMSD plot of MD simulation 20ns trajectory obtained for 'Quercetin-PAI-1' complex

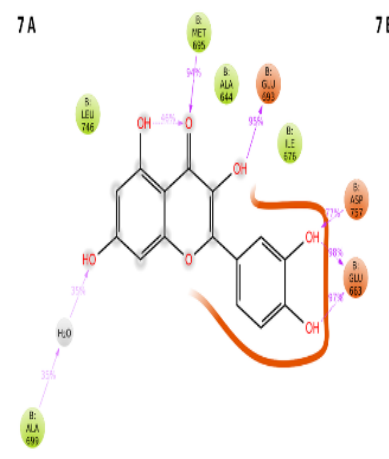

$7 B$

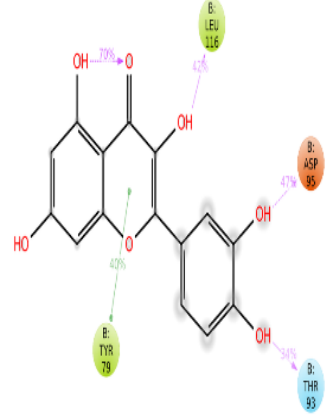

Figure 7: Amino acids involved in molecular interaction for more than $30 \%$ of the total simulation time (7A) Quercetin and EphA2 (7B) Quercetin and PAl-1

To identify the binding mode of quercetin with the active sites of EphA2 and PAl-1, atomic level information about interactive forces involved is mandatory. Schematic details of quercetin interaction with EphA2 residues predicted over the 20ns simulation studies are shown in Figure 7A. Here, the interactions that retained for more than $30 \%$ of the total simulation time were selected. Quercetin showed strong hydrogen bonding with Glu663, Glu693, Met695 and Asp757 amino acid residues of EphA2, however, Glu663 interaction with quercetin was observed for the entire simulation time. Moreover, Ala699 (35\%) showed water bridge interaction and Leu746, Ala644 and Ile676 residues showed hydrophobic interactions with quercetin. On the other hand, quercetin and $\mathrm{PAl}-1$ interaction over the 20ns simulation are shown in Figure 7B. Quercetin interacted with Tyr79, Thr93, Asp95 and Leu116 amino acid residues of PAl-1 for more than $30 \%$ of the simulation time. Tyr79 (40\%) showed a $\pi-\pi$ interaction with quercetin, while, Thr93 (34\%), Asp95 (47\%) and Leu116 (42\%) interacted via hydrogen bonding.

\section{DISCUSSION}

Ascertaining new-targeted efficacious treatments for gliomas presents an immense challenge. In the present study, EGFR, EphA2, NMRPTase and PAl-1 as gliomas target proteins were chosen, and their active sites were docked with quercetin. Various mechanistic investigations have revealed the multi-targeting anticancer potency of quercetin via interacting with cell cycle regulatory proteins, activate p53 and caspases, PI3K inhibition and cytochrome c release $[6,7]$.

Several oncogenic drivers for example receptor tyrosine kinases (RTKs) are involved in glioblastoma tumour development, and therapeutic resistance have been identified through genomic and proteomic analyses such as EGFR. Importantly, EGFR expression is extremely low or even undetectable in normal brain tissues, whereas, it has been found to be the most commonly amplified gene in glioblastoma [12]. The profound role of EGFR as the most vital factor in promoting gliomas has fascinated several inquiries into EGFR inhibition targeted at inducing/promoting tumour cell apoptosis or increasing its sensitivity to potential adjuvant therapies. Notably, in the present study, quercetin bound via hydrogen bonding to one of the important amino acids, i.e., Lys745 of ATP binding pocket of EGFR. Similarly, Shen et al [13] also observed hydrogen bonding of lycorine to Lys745 residue of EGFR. Moreover, quercetin interaction with important amino acid residues of 'ATP binding pocket' of EGFR and negative $\triangle G$ suggested that quercetin might function as a competitive inhibitor for ATP binding with EGFR.

The second target for the present study was EphA2. Proliferating adult epithelial cells express EphA2 receptors on their surfaces unlike other Eph receptors, which are present during embryonic development in the nervous system [14,15]. However, previous studies have revealed that overexpression of EphA2 is significantly associated with many human epithelial malignancies including colon [16], breast [16] and prostate [17] carcinomas. This increase in EphA2 levels in cancer might be attributed due to a generally reduced 
ligand-mediated degradation [15] as reported in unstable intercellular contacts in a cancer tissue that may hamper EphA2 receptor ability of interacting with ephrinA1 ligand present on adjoining cells [18]. These evident characteristics and associations make EphA2 an ultimate candidate for targeting several cancers [14]. Thus, similar to EGFR, lowlevel expression in normal brain tissues and high levels in glioblastomas makes EphA2 a potential molecular target for therapeutic intervention. Based on the present study assessment, quercetin showed strong interaction towards EphA2 in terms of binding energy. Co-crystallized ligand phosphoaminophosphonic acid-adenylate ester existing in the three-dimensional structure (PDB:1MQB) formed hydrogen bonding with Thr692 and Met695 amino acid residues of EphA2. Interestingly, quercetin also showed hydrogen-bonding interaction with these two important amino acid residues of active site of EphA2. In addition, Kirubakaran et al also showed the interaction of tetracenomycin $D$ and chartreusin with Met695 residue of EphA2 [19].

Third target for the present study was NMPRTase. Nicotinamide Adenine Dinucleotide (NAD+) biosynthesis has been found to be activated in various cancers. The salvage pathway of NAD+ requires transformation of nicotinamide mononucleotide (NMN) from free nicotinamide, an important step catalyzed by NMPRTase. Moreover, several fold upregulated expression of NMPRTase was observed during microarray analyses of grade II to IV glioma cells in comparison to normal cells [20]. In the present study, quercetin interacted with important amino acids such as F193, Val356, Gly381 and Lys389 of NMPRTase 'active site'.

Interestingly, nicotinamide ribose monophosphate (control) showed similar interaction with the amino acids Val356, Gly381 and Lys389 of NMPRTase via hydrogen bonds [21]. In addition, most of the novel screened compounds have also shown interaction with $\mathrm{F} 193$ amino acid of the binding pocket of NMRPTase [21]. Similarity in interactions with crucial amino acids of NMRPTase supported the present findings with quercetin.

The final and fourth target of the present study was PAI-1. Plasminogen activator inhibitor (PAl-1) regulates angiogenesisinduced tumor growth, and involved in the adhesion, migration and invasion of cancer cells [22]. Glioblastomas expresses a considerable amount of PAI-1, and PAl-1 increased expression was often associated with metastasis of the tumour and poor clinical outcomes [23]. According to a 2013 study, the two pockets of PAI-1 containing Ala12, Tyr37, Gly38, Ala40, Ser41, Met62, Phe64, Leu75, Thr93, Thr94, Asp95 and Asn167 amino acid residues play an important role in drug design, and specifically, Ser41 is crucial bridge between these two pockets [24]. Interestingly, the present results also showed interaction of quercetin with some of these amino acid residues (Ser41, Leu75, Thr93, Thr94 and Asp95) of PAl-1.

Overall, molecular docking outcomes demonstrated that quercetin showed strong interaction with active sites of two most promising targets (EphA2 and PAl-1) of gliomas. However, molecular docking analysis provides only a static perspective on the docked complex, while, MD simulation estimates the atomic movements with respect to the time by incorporating Newton's equation of motion [25]. Thus, stability of the docked complex was analyzed by MD simulation study. Minimal fluctuations were observed in RMSD analysis during MD simulation that directly correlates to the stability of both 'quercetin and EphA2' and 'quercetin and PAI-1' complexes. Moreover, wet lab experiments are needed to establish quercetin as a potent therapeutic agent for gliomas. However, computational predictions set the base for further research and its results often relate well with in vitro experiments. At the end, it can be safely stated that quercetin has vast potential and its scaffolds could be utilized to develop therapeutic agents against gliomas in near future.

\section{CONCLUSION}

Treatment of gliomas is one of the biggest challenges for the medical community worldwide. Thus, exploration of alternative therapy to copeup with current therapeutic challenges is the urgent need of time. The present study revealed that quercetin interacted with crucial amino acid residues of the active site of the emerging gliomas targets, i.e., epidermal growth factor receptor (EGFR), ephrin type-A receptor 2 (EphA2), nicotinamide phosphoribosyltransferase (NMRPTase) and plasminogen activator inhibitor-1 (PAI-1). Molecular docking interaction showed that quercetin interacted strongly with EphA2 and PAl-1, while, simulation analysis showed that these interactions between 
'quercetin and EphA2' and 'quercetin and PAI-1' were stable too. These predictive evaluations are important to comprehend the biochemical interaction to get insight of the mechanistic aspects of quercetin and to design new potent therapeutic agents based on quercetin scaffolds against gliomas.

\section{DECLARATIONS}

\section{Acknowledgement}

The authors acknowledge with thanks Research Deanship, University of Hail, Hail, Kingdom of Saudi Arabia for providing financial support under Project no. RG-191325.

\section{Conflict of interest}

No conflict of interest is associated to this work.

\section{Contribution of authors}

We declare that this work was done by the authors named in this article and all liabilities pertaining to claims relating to the content of this article will be borne by the authors. SMDR, TH and KM participated in molecular docking, Ligplot analysis and compilation of data. AM, ASA and GMS participated in molecular simulation analysis, data analysis and writing of MS.

\section{Open Access}

This is an Open Access article that uses a funding model which does not charge readers or their institutions for access and distributed under the terms of the Creative Commons Attribution License (http://creativecommons.org/licenses/by/ 4.0) and the Budapest Open Access Initiative (http://www.budapestopenaccessinitiative.org/rea d), which permit unrestricted use, distribution, and reproduction in any medium, provided the original work is properly credited.

\section{REFERENCES}

1. Thakkar JP, Dolecek TA, Horbinski C, Ostrom QT, Lightner DD, Barnholtz-Sloan JS, Villano JL. Epidemiologic and molecular prognostic review of glioblastoma. Cancer Epidemiology and Prevention Biomarkers 2014; 23(10): 1985-1996.

2. Minniti G, De Sanctis V, Muni R, Filippone F, Bozzao A, Valeriani M, Osti MF, De Paula U, Lanzetta G, Tombolini V, Enrici RM. Radiotherapy plus concomitant and adjuvant temozolomide for glioblastoma in elderly patients. Journal of Neuro-oncology 2008; 88(1): 97103.
3. Lei CS, Hou YC, Pai MH, Lin MT, Yeh SL. Effects of quercetin combined with anticancer drugs on metastasis-associated factors of gastric cancer cells: in vitro and in vivo studies. The Journal of Nutritional Biochemistry 2018; 51: 105-113.

4. Ward AB, Mir H, Kapur N, Gales DN, Carriere PP, Singh S. Quercetin inhibits prostate cancer by attenuating cell survival and inhibiting anti-apoptotic pathways. World Journal of Surgical Oncology 2018; 16(1) :1-2.

5. Sadhukhan P, Kundu M, Chatterjee S, Ghosh N, Manna $P$, Das J, Sil PC. Targeted delivery of quercetin via $\mathrm{pH}$ responsive zinc oxide nanoparticles for breast cancer therapy. Materials Science and Engineering: C 2019; 100: $129-140$

6. Wu $Q$, Needs $P W, L u Y$, Kroon $P A$, Ren $D$, Yang $X$. Different antitumor effects of quercetin, quercetin-3'sulfate and quercetin-3-glucuronide in human breast cancer MCF-7 cells. Food \& Function 2018; 9(3): 17361746.

7. Jia L, Huang S, Yin X, Zan Y, Guo Y, Han L. Quercetin suppresses the mobility of breast cancer by suppressing glycolysis through Akt-mTOR pathway mediated autophagy induction. Life Sciences 2018; 208: 123-130.

8. da Silva $A B$, Coelho PL, das Neves Oliveira M, Oliveira $J L$, Amparo JA, da Silva KC, Soares JR, Pitanga BP, dos Santos Souza C, de Faria Lopes GP, da Silva VD. The flavonoid rutin and its aglycone quercetin modulate the microglia inflammatory profile improving antiglioma activity. Brain Behavior, and Immunity 2020; 85: 170185.

9. Lesjak M, Beara I, Simin N, Pintać D, Majkić T, Bekvalac $K$, Orčić D, Mimica-Dukić N. Antioxidant and antiinflammatory activities of quercetin and its derivatives. Journal of Functional Foods 2018; 40: 68-75.

10. Rizvi SM, Shakil S, Haneef M. A simple click by click protocol to perform docking: AutoDock 4.2 made easy for non-bioinformaticians. Excli Journal 2013; 12: 831.

11. Laskowski RA, Swindells MB. LigPlot+: multiple ligandprotein interaction diagrams for drug discovery., Journal of Chemical Information and Modeling 2011; 51: 27782786.

12. Gollapalli K, Ghantasala S, Atak A, Rapole S, Moiyadi A, Epari S, Srivastava S. Tissue proteome analysis of different grades of human gliomas provides major cues for glioma pathogenesis. OMICS: A Journal of Integrative Biology 2017; 21(5): 275-84.

13. Shen J, Zhang T, Cheng Z, Zhu N, Wang H, Lin L, Wang $\mathrm{Z}$, Yi H, Hu M. Lycorine inhibits glioblastoma multiforme growth through EGFR suppression. Journal of Experimental \& Clinical Cancer Research. 2018; 37(1): 157.

14. Andres AC, Reid HH, Zürcher G, Blaschke RJ, Albrecht $D$, Ziemiecki A. Expression of two novel eph-related receptor protein tyrosine kinases in mammary gland development and carcinogenesis. Oncogene 1994; 9(5): 1461

15. Kinch MS, Carles-Kinch K. Overexpression and functional alterations of the EphA2 tyrosine kinase in

Trop J Pharm Res, April 2021; 20(4): 822 
cancer. Clinical \& Experimental metastasis 2003; 20(1): 59-68.

16. Ogawa $K$, Pasqualini $R$, Lindberg RA, Kain R, Freeman $A L$, Pasquale EB. The ephrin-A1 ligand and its receptor, EphA2, are expressed during tumor neovascularization. Oncogene 2000; 19(52): 6043-6052.

17. Walker-Daniels J, Coffman K, Azimi M, Rhim JS, Bostwick DG, Snyder P, Kerns BJ, Waters DJ, Kinch MS. Overexpression of the EphA2 tyrosine kinase in prostate cancer. The Prostate 1999; 41(4): 275-80.

18. Zantek ND, Azimi M, Fedor-Chaiken M, Wang B, Brackenbury $R$, Kinch MS. E-cadherin regulates the function of the EphA2 receptor tyrosine kinase. Cell Growth and Differentiation-Publication American Association for Cancer Research 1999; 10(9): 629-38.

19. Kirubakaran $P$, Kothapalli $R$, Singh $K D$, Nagamani $S$, Arjunan S, Muthusamy K. In silico studies on marine actinomycetes as potential inhibitors for Glioblastoma multiforme. Bioinformation 2011; 6(3): 100.

20. Reddy PS, Umesh S, Thota B, Tandon A, Pandey $P$, Hegde AS, Balasubramaniam A, Chandramouli BA, Santosh $V, \quad S$ Rao MR, Kondaiah $P$.
PBEF1/NAmPRTase/Visfatin: a potential malignant astrocytoma/glioblastoma serum marker with prognostic value. Cancer Biology \& Therapy 2008; 7(5): 663-8.

21. Chandra N, Bhagavat $R$, Sharma $E$, Sreekanthreddy $P$, Somasundaram K. Virtual screening, identification and experimental testing of novel inhibitors of PBEF1/Visfatin/NMPRTase for glioma therapy. Journal of Clinical Bioinformatics 2011; 1(1): 5.

22. Li S, Wei X, He J, Tian X, Yuan S, Sun L. Plasminogen activator inhibitor-1 in cancer research. Biomedicin Pharmacother 2018; 105: 83-94.

23. Kit OI, Frantsiyants EM, Kozlova LS, Rostorguev EE, Balyazin-Parfenov IV, Pogorelova YA. A plasminogen regulation system in brain tumors. Zh Vopr Neurokhir im NN Burdenko 2017; 81(2): 22-27.

24. Lin Z, Jensen JK, Hong Z, Shi X, Hu L, Andreasen PA, Huang M. Structural insight into inactivation of plasminogen activator inhibitor-1 by a small-molecule antagonist. Chem Biol 2013; 20(2): 253-61.

25. Adcock SA, McCammon JA. Molecular dynamics: survey of methods for simulating the activity of proteins. Chem Rev 2006; 106(5): 1589-615. 